\title{
Sterol Hormone 20E Regulates Autophagy via Multiple Pathways in Insect
}

\author{
Wenmei Wu${ }^{1}$, Yichen Dai ${ }^{1}$, Kang $\mathrm{Li}^{2}$ and Ling Tian ${ }^{1 *}$ \\ ${ }^{1}$ Guangdong Provincial Key Laboratory of Agro-animal Genomics and Molecular Breeding/Guangdong Provincial Sericulture and Mulberry \\ Engineering Research Center, South China Agricultural University, China \\ ${ }^{2}$ Guangdong Provincial Key Laboratory of Insect Developmental Biology and Applied Technology, Institute of Insect Science and Technology, South \\ China Normal University, China
}

Submission: July 01, 2019; Published: July 11, 2019

*Corresponding author: Ling Tian, Professor, College of Animal Science, South China Agricultural University, Guangzhou, China

Keywords: 20E; Induction of autophagy; Multiple pathways; Insects

\section{Mini Review}

Sterol hormone 20-hydroxyecdysone (20E), specifically biosynthesized from cholesterol under the catalysis of a series of cytochrome P450 enzymes, is pivotal for insect molting and metamorphosis. Ecdysone, the precursor of $20 \mathrm{E}$, is synthesized and secreted by a pair of prothoracic glands in holometabolous insects, and then is released into the hemolymph and converted to the active form 20E in peripheral tissues such as fat body, midgut and malpighian tubes during larval stage [1]. In the adults of some insects, the presence of ecdysone in the ovary was recognized more than 40 years ago, and now it is well known that ovarian follicular cells produce ecdysone from scratch [2]. However, in some Lepidoptera, male gonads also release in moderately large amounts of ecdysone in vitro [3] .

Via binding its receptor complex EcR-USP, 20E rapidly and highly induced the expression of primary-response genes including nuclear receptors or transcription factors such as $E 75$, $E 93, B r-C$, and E74, which are requisite for insect larval-pupal metarmorphosis [4-6]. 20E massively induced the expression of three different BmE75 isofoms in Bombyx, which consequentially coordinated feedback to 20E biosynthesis [4]. E93 is encoded by a member of the helix-turn-helix (HTH) transcription factor family and is involved in the crosstalk of $20 \mathrm{E}$ signaling with juvenile hormone $(\mathrm{JH})$ signaling via JH primary response gene Kr-h1 [5]. Notably, 20E signaling predominantly mediates the occurrence of autophagy in larval tissue and organs during larval molting and larval-pupal metamorphosis [7].

In Drosophila, a peak of $20 \mathrm{E}$ at the end of the larval period triggers metamorphosis accompanying with elimination of the larval tissues, which are no longer necessary for adults, and to recycle molecular materials for the formation of adult tissues [2]. Moreover, 20E inhibits the PI3K/mTOR signaling to activate ATG1/ATG13 complex to initiate autophagosome formation in Drosophila fat body [8]. 20E is necessary and sufficient to induce autophagy in larval tissue via upregulating the expression of several autophagy related (Atg) genes, implying ecdysoneinduced autophagy as a crucial process in degradation of larval tissues [9]. Furthermore, 20E signaling activates E93 to induce autophagy in Drosophila; in contrast, mutation of E93 blocks the destruction of the larval fat body via autophagy [5].

In Bombyx, 20E signaling is also found to upregulate autophagy through induction of most of all Atg gene expressions and inhibition of mTOR pathway. Moreover, the early-response transcription factors of $20 \mathrm{E}$ (i.e. Br-C, E74, E75, and E93) involved in its regulation on autophagy [10]. Bombyx E75 was showed to be indispensable for autophagy induction during larval-pupal metamorphosis [4]. Notably, the 20E primary response genes E93, which acts through GAGA-containing motifs, modulates 20E-EcR-USP to promote larval tissue remodeling and adult tissue formation during Bombyx larval-pupal metamorphosis [6]. In addition, 20E induces starvation-like condition in Bombyx by reducing food consumption to trigger autophagy [10]. Our previous work displayed that being cleaved into BmATG5 and BmATG6, BmATG5-tN and BmATG6-C mediated the switching between autophagy and apoptosis induced by their common stimuli 20E and starvation in Bombyx [11,12]. 20E is also reported to regulate autophagy beyond induction of Atg genes transcriptions and inhibition of mTOR signaling. In Helicoverpa armigera, 20E regulates the conjugation of ATG12-ATG5 in a concentration and time-dependent manner to promote insect 
midgut programmed cell death; moreover, 20E induces an increase intracellular calcium levels, and thereby switches autophagic cell survival to apoptotic cell death $[13,14]$.

In addition, our previous studies found that $20 \mathrm{E}$ also induced lysosome acidification [10]; nevertheless, the precise mechanism is now well documented. Our recent works showed that the developmental profiles of all Bombyx V-ATPases, which were responsible for pumping protons across the plasma membranes in numerous types of eukaryotic cells, were in accordance with $20 \mathrm{E}$ titer in the hemolymph during larvalpupal metamorphosis. 20E treatment induced V-ATPase gene expression through the activation of transcription factor $E B$ and the subcomplex assembly of BmV-ATPases via promoting the interaction between different subunits. In contrast, RNAi of the $20 \mathrm{E}$ receptor gene $B m U s p$ reduced both transcription and translation of $V$-ATPases (in submission). Besides, we also found that $20 \mathrm{E}$ dephosphorylated the histone deacetylase BmRpd3, which mediates the deacetylation modification of ATG proteins, and resulted in its nucleo-cytoplasmic translocation and consequent promotion of autophagy occurrence (in submission). In summary, 20E signaling upregulates autophagy occurrence through multiple pathways including induction of Atg and V-ATPases gene expression, inhibition of mTOR pathway, and deacetylation modification of ATG proteins. For the multifarious functions of the sterol hormone 20E signaling, its deep physiological functions and the precise mechanism on autophagy induction is worthy for further investigation.

\section{Acknowledgments}

This study was supported by the National Science Foundation of China (grants 31472042 and 31672368 to LT), Natural Science Foundation of Guangdong Province (grant 2017A030311024 to LT).

\section{References}

1. Kefi M, Balabanidou V, Douris V, Lycett G, Feyereisen R, et al. (2019) Two functionally distinct CYP4G genes of Anopheles gambiae contribute to cuticular hydrocarbon biosynthesis. Insect Biochem Mol Biol 110: 52-59.

1. Engelmann F (1971) 20-hydroxyecdysone, what it can do. Science 174(4013): 1041.
2. Horike N, Sonobe H (1999) Ecdysone 20-monooxygenase in eggs of the silkworm, Bombyx mori: enzymatic properties and developmental changes. Arch Insect Biochem Physiol 41(1): 9-17.

3. Li K, Tian L, Guo Z, Guo S, Zhang J, et al. (2016) 20-Hydroxyecdysone (20E) Primary Response Gene E75 Isoforms Mediate Steroidogenesis Autoregulation and Regulate Developmental Timing in Bombyx. J Biol Chem 291(35): 18163-18175.

4. Liu H, Wang J, Li S (2014) E93 predominantly transduces 20-hydroxyecdysone signaling to induce autophagy and caspase activity in Drosophila fat body. Insect Biochem Mol Biol 45: 30-39.

5. Liu X, Dai F, Guo E, Li K, Ma L, et al. (2015) 20-Hydroxyecdysone (20E) Primary Response Gene E93 Modulates 20E Signaling to Promote Bombyx Larval-Pupal Metamorphosis. J Biol Chem 290(45): 2737027383.

6. Tian L, Guo E, Wang S, Liu S, Jiang RJ, et al. (2010) Developmental regulation of glycolysis by 20-hydroxyecdysone and juvenile hormone in fat body tissues of the silkworm, Bombyx mori. J Mol Cell Biol 2(5): 255-263.

7. Rusten TE, Lindmo K, Juhasz G, Sass M, Seglen PO, et al. (2004) Programmed autophagy in the Drosophila fat body is induced by ecdysone through regulation of the PI3K pathway. Dev Cell 7(2): 179192.

8. Yin VP, Thummel CS (2005) Mechanisms of steroid-triggered programmed cell death in Drosophila. Semin Cell Dev Biol 16(2): 237243.

9. Tian L, Ma L, Guo E, Deng X, Ma S, et al. (2013) 20-Hydroxyecdysone upregulates Atg genes to induce autophagy in the Bombyx fat body. Autophagy 9(8): 1172-1187.

10. Xie K, Tian L, Guo X, Li K, Li J, et al. (2016) BmATG5 and BmATG6 mediate apoptosis following autophagy induced by 20-hydroxyecdysone or starvation. Autophagy 12(2): 381-396.

11. Romanelli D, Casartelli M, Cappellozza S, de Eguileor M, Tettamanti G (2016) Roles and regulation of autophagy and apoptosis in the remodelling of the lepidopteran midgut epithelium during metamorphosis. Sci Rep 6: 32939.

12. Li YB, Li XR, Yang T, Wang JX, Zhao XF (2016) The steroid hormone 20-hydroxyecdysone promotes switching from autophagy to apoptosis by increasing intracellular calcium levels. Insect Biochem Mol Biol 79: 73-86.

13. Li YB, Yang T, Wang JX, Zhao XF (2018) The Steroid Hormone 20-Hydroxyecdysone Regulates the Conjugation of Autophagy-Related Proteins 12 and 5 in a Concentration and Time-Dependent Manner to Promote Insect Midgut Programmed Cell Death. Front Endocrinol (Lausanne) 9: 28.

Your next submission with Juniper Publishers will reach you the below assets

- Quality Editorial service

- Swift Peer Review

- Reprints availability

- E-prints Service

- Manuscript Podcast for convenient understanding

- Global attainment for your research

- Manuscript accessibility in different formats

( Pdf, E-pub, Full Text, Audio)

- Unceasing customer service

Track the below URL for one-step submission https://juniperpublishers.com/online-submission.php 